\title{
Relationship between 17-hydroxyprogesterone caproate concentrations and gestational age at delivery in twin gestation
}

\author{
Steve N. CARITIS, MD ${ }^{1}$, Hyagriv N. SIMHAN, MD ${ }^{1}$, Yuan ZHAO, MS ${ }^{2}$, Dwight J. ROUSE, \\ MD $^{3}$, Alan M. PEACEMAN, MD ${ }^{4}$, Anthony SCISCIONE, DO ${ }^{5}$, Catherine Y. SPONG, MD ${ }^{6}$, \\ Michael W. VARNER, MD ${ }^{7}$, Fergal D. MALONE, MD ${ }^{8}$, Jay D. IAMS, MD $^{9}$, Brian M. MERCER, \\ MD $^{10}$, John M THORP Jr, MD ${ }^{11}$, Yoram SOROKIN, MD ${ }^{12}$, Marshall CARPENTER, MD ${ }^{13}$, Julie \\ LO, MD ${ }^{14}$, Susan M. RAMIN, MD ${ }^{15}$, Margaret HARPER, MD, MS ${ }^{16}$, and for the Eunice \\ Kennedy Shriver National Institute of Child Health and Human Development Maternal-Fetal \\ Medicine Units Network \\ ${ }^{1}$ Department of Obstetrics, Gynecology and Reproductive Sciences University of Pittsburgh \\ School of MedicinePittsburgh, PA \\ ${ }^{2}$ The George Washington University Biostatistics Center, Washington, DC \\ ${ }^{3}$ Departments of Obstetrics and Gynecology of the University of Alabama at Birmingham, \\ Birmingham, AL \\ ${ }^{4}$ Northwestern University, Chicago, IL \\ ${ }^{5}$ Drexel University, Philadelphia, PA \\ ${ }^{6}$ the Eunice Kennedy Shriver National Institute of Child Health and Human Development, \\ Bethesda, MD \\ ${ }^{7}$ University of Utah, Salt Lake City, UT \\ ${ }^{8}$ Columbia University, New York, NY \\ ${ }^{9}$ The Ohio State University, Columbus, $\mathrm{OH}$ \\ ${ }^{10}$ Case Western Reserve University-MetroHealth Medical Center, Cleveland, $\mathrm{OH}$ \\ ${ }^{11}$ University of North Carolina at Chapel Hill, Chapel Hill, NC \\ ${ }^{12}$ Wayne State University, Detroit, MI \\ ${ }^{13}$ Brown University, Providence, RI \\ ${ }^{14}$ University of Texas Southwestern Medical Center, Dallas, TX \\ ${ }^{15}$ University of Texas Health Science Center at Houston, Houston, TX \\ ${ }^{16}$ Wake Forest University Health Sciences, Winston-Salem, NC
}

\footnotetext{
(C) 2012 Mosby, Inc. All rights reserved.

Corresponding Author: Steve N. Caritis, MD, 300 Halket Street, Room 2229, Pittsburgh, PA 15213, Business Phone: 412-641-4874, Home Phone: 412-362-0980, Fax Number: 412-641-1133.

DISCLOSURE: None of the authors have a conflict of interest

Reprints not available

Publisher's Disclaimer: This is a PDF file of an unedited manuscript that has been accepted for publication. As a service to our customers we are providing this early version of the manuscript. The manuscript will undergo copyediting, typesetting, and review of the resulting proof before it is published in its final citable form. Please note that during the production process errors may be discovered which could affect the content, and all legal disclaimers that apply to the journal pertain.
} 


\section{Abstract}

Objective-To evaluate in women with twin gestation the relationship between 17hydroxyprogesterone caproate (17-OHPC) concentration and gestational age at delivery and select biomarkers of potential pathways of drug action.

Study Design-Blood was obtained between 24-28 weeks (epoch 1) and 32-35 weeks (epoch 2 ) in 217 women with twin gestation receiving 17-OHPC or placebo. Gestational age at delivery and concentrations of 17-OHPC, 17-hydroxyprogesterone, progesterone, C-reactive protein (CRP) and corticotrophin releasing hormone were assessed.

Results-Women with higher concentrations of 17-OHPC delivered at earlier gestational ages than women with lower concentrations $(\mathrm{p}<0.001)$. Women receiving 17-OHPC demonstrated significantly higher $(\mathrm{p}=0.005)$ concentrations of CRP in epoch 1 than women receiving placebo but CRP values were similar in epoch 2 in both groups. A highly significant $(p<0.0001)$ positive relationship was observed between 17-OHPC concentration and progesterone and 17hydroxyprogesterone concentrations at both epochs. CRH concentrations did not differ by treatment group.

Conclusion-17-OHPC may adversely impact gestational age at delivery in women with twin gestation.

\section{Keywords}

17-hydroxyprogesterone caproate (17-OHPC); pharmacodynamics; twin gestation

\section{Introduction}

17-hydroxyprogesterone caproate (17-OHPC) reduces the rate of preterm birth in women with a singleton gestation and a prior preterm birth. ${ }^{1}$ Despite widespread clinical use of this agent the mechanism of action and target organ of this therapy are not known. Knowledge of the mechanism of action and target tissue(s) of this therapy would enable a better understanding of preterm labor, its etiology and possible development and refinement of prevention strategies. The purpose of this study was to define in a cohort of women with twin gestation the relationship between 17-OHPC concentration and gestational age at delivery. These women were participants in a placebo - controlled randomized clinical trial to evaluate the efficacy of 17-OHPC in preventing preterm birth. ${ }^{2}$ Although treatment proved ineffective in twins, blood samples collected during 17-OHPC treatment allowed an evaluation of the relationship between plasma drug concentration and gestational age at delivery. Additionally we evaluated the relation between plasma 17-OHPC concentration and select gestational hormones as well as biomarkers of other potential pathways of drug action, C-reactive protein (CRP) as a marker of systemic inflammation and Corticotropin Releasing Hormone (CRH) as a marker of the endocrine pathway involved in human parturition. ${ }^{3,4}$

\section{MATERIALS AND METHODS}

\section{Patients and Drug Administration}

This was a planned ancillary study to the MFMU Network randomized trial of women with twins who were receiving either intramuscular 17-hydroxyprogesterone caproate or placebo. ${ }^{2}$ A total of 661 women with twins were recruited for the randomized trial. Subjects received masked weekly injections of either $250 \mathrm{mg} 17-\mathrm{OHPC}$ in $1 \mathrm{ml}$ castor oil or $1 \mathrm{ml}$ castor oil alone from the time of enrollment (16 0/7 weeks - 20 6/7 weeks) until 34 weeks 6 days or until delivery, whichever came first. 


\section{Blood Sampling Schedule}

Consenting subjects were informed that two blood samples would be taken, one between 24-28 weeks (epoch 1) and one between 32-35 weeks (epoch 2) for determination of progesterone, 17-hydroxyprogesterone, 17-OHPC, CRP and CRH concentrations. The timing of epoch 1 was intended to obtain a blood sample after a minimum of four injections would have been administered to allow steady state to be reached. The timing of the second epoch was intended to evaluate gestational age related changes in pharmacodynamic parameters.

\section{Sample Analysis}

For measurement of 17-OHPC, progesterone, 17-hydroxyprogesterone and CRP, blood was collected in $10 \mathrm{ml}$ tubes with anticoagulant and centrifuged within one hour at $2500 \mathrm{rpm}$ for 10 minutes. The supernatant plasma was aliquoted to $1 \mathrm{ml}$ tubes and was frozen at $-70^{\circ} \mathrm{C}$ until analysis. Quantification of 17-OHPC, progesterone and 17-hydroxyprogesterone concentration was performed using high performance liquid chromatography with tandem mass spectrometry (LC-MS). The assay methodology has been reported elsewhere. ${ }^{5}$ The lower assay limit of detection for 17-OHPC was $1 \mathrm{ng} / \mathrm{ml}$, inter and intra assay variability at $10 \mathrm{ng} / \mathrm{ml}$ was $7.9 \%$ and $5.2 \%$ respectively. For progesterone and 17-hydroxyprogesterone, the lower limit of detection were $2 \mathrm{ng} / \mathrm{ml}$ and $1 \mathrm{ng} / \mathrm{ml}$ respectively. The inter and intra assay variabilities for progesterone $(5 \mathrm{ng} / \mathrm{ml})$ were 7.7 and 8.4 percent respectively and 12.3 and 9.5 percent for 17-hydroxyprogesterone. At the time of the analysis, the analyst and the clinical centers involved in recruitment were blinded to the treatment assignment.

CRP in maternal plasma was measured with a commercially available ELISA kit (Alpha Diagnostic International, San Antonio, TX), with a coefficient of variation of 3.0\%. Separate linear standard curves were generated for each sample plate, and those values that were greater than the maximum concentration of the standard were repeated after progressive dilutions.

For CRH measurements, blood was collected in a $5 \mathrm{ml}$ chilled tube with EDTA and aprotinin $\left(500 \mathrm{KIU} / \mathrm{ml}\right.$ blood), centrifuged at $2500 \mathrm{rpm}$ for 10 minutes at $4^{\circ} \mathrm{C}$. Aliquots of the supernatant were then transferred to a polypropylene tube then stored at $-70^{\circ} \mathrm{C}$ until analyzed. CRH peptide was extracted from plasma using Linton's method. ${ }^{6}$ The dried extracts were resuspended in $0.5 \mathrm{ml}, 0.05 \mathrm{~mol} / \mathrm{L}$ phosphate buffer, $\mathrm{pH} 7.3$, containing $5.8 \mathrm{~g} / \mathrm{L}$ $\mathrm{NaCl}, 9.5 \mathrm{~g} / \mathrm{L}$ ethylenediamine tetraacetate, $1.0 \mathrm{~g} / \mathrm{L} \mathrm{NaN3}$, and $1 \mathrm{~mL} / \mathrm{L}$ Triton and transferred to a storage vial. Vials were rinsed with another $0.5 \mathrm{ml}$ buffer to recuperate residual peptide. Pooled aliquots of the reconstituted extracts were stored at $-80^{\circ} \mathrm{C}$ and assayed in batches. Each methanol extraction consisted of spiked plasma controls from a healthy male donor. Purified, CRH peptide (Peninsula Laboratories) was adjusted to $5 \mathrm{ng} / \mathrm{ml}$ and $2.5 \mathrm{ng} / \mathrm{ml}$ in the donor plasma to serve as both assay controls and as an extraction reference standard. Because methanol extraction efficiency varies from batch to batch, all CRH test sample values were adjusted accordingly. For example, the $5 \mathrm{ng}$ control following extraction was tested at $4.5 \mathrm{ng}$ CRH (an extraction efficiency of 90\%, assuming the detection method is complete). In this case, all test samples would be adjusted up by a factor of 1.11 in this batch to compensate for incomplete $\mathrm{CRH}$ extraction.

For the enzyme immunoassay, EIA, (Peninsula Laboratories, San Carlos, CA), anti-CRH antibodies were plated on a 96-well plate. In each well, a known concentration of biotinylated tracer was co-incubated for 2 hours at $21-23^{\circ} \mathrm{C}$ with anti-human $\mathrm{CRH}$ and 50ul of sample. Following a wash step, streptavidin-conjugated horseradish peroxidase was added to each well and the incubation continued for 1 hour. The wells were again washed and the substrate, tetramethyl benzidine dihydrochloride was added. After 45 minutes, the 
reaction was stopped with $2 \mathrm{~N} \mathrm{HCL}$ and the absorbance was read at $450 \mathrm{~nm}$. The data were plotted against a CRH standard S-shaped curve on a semi-log plot, which was provided as an Excel (Microsoft, Redmond, WA) spreadsheet by the Bachem Group, a member of Peninsula Labs. In our laboratory, the intra and inter-assay variability of the CRH EIA is comparable to other commercial immunoassay kits, $\unlhd 0 \%$.

\section{Statistical Analysis}

For all analyses, we excluded women who did not receive all of their scheduled injections up to the time of the first blood draw (epoch 1). For analyses involving data from the second blood draw we also excluded women who did not receive all of their injections between the first and second blood draw. For analyses related to gestational age at delivery, we excluded all women who did not receive all their scheduled injections up to the time of delivery or until 35 weeks gestation. Rate of change for each analyte was calculated as the difference between the first and second epoch value divided by time elapsed in weeks between blood draws. Chi-square and Wilcoxon tests were used to make univariable two-group comparisons for categorical and continuous variables respectively. Spearman rank correlation coefficients were calculated to assess the pairwise correlation between continuous variables. Proportional hazards regression was used to analyze the relationship between the analytes and gestational age at delivery, adjusting for gestational age at the time of blood draw, as well as for race/ethnicity and BMI which may impact plasma 17-OHPC concentrations (7). Indicated deliveries were censored at the time of delivery. For the multivariable analysis, monochorionic-diamniotic pregnancies were excluded because placentation, progesterone levels and endogenous steroid hormones could affect gestational age at delivery compared with dichorionic-diamniotic pregnancies. For the proportional hazards models assessing gestational age at delivery versus CRP and versus progesterone, 17-OHPC was also included as a covariate because 17-OHPC concentration increases with gestational age as a consequence of greater number of castor oil depots seen with weekly injections. Generalized R-squared values were calculated to estimate the proportion of explained variation. Scatter plots were used to demonstrate relationships visually. For all statistical tests, nominal two-sided p-values are reported with statistical significance defined as a p-value $<0.05$. No corrections were made for multiple comparisons. SAS Software (SAS Institute, Cary, North Carolina) was used for these analyses.

\section{RESULTS}

\section{Characteristics of the Study Population}

Among the 661 subjects enrolled in the main trial, 217 agreed to the study, received all scheduled injections and had blood drawn at epoch 1;118 in the placebo group and 99 in the 17-OHPC group. Table 1 compares demographic parameters of these subjects at enrollment according to treatment group. Gestational age at delivery is also provided. There were no significant differences between women treated with 17-OHPC or placebo. A total of 92 of these women in the placebo group and 72 in the 17-OHPC group had received all of their injections and had a blood drawn by epoch 2 . A median of seven injections had been administered by epoch 1 and 14 by epoch 2 in each of the two groups. The gestational age at which blood samples were obtained was similar in the two treatment groups at the first sampling but there was a significant $(\mathrm{p}=0.04)$ difference of four days in gestational age at the second blood sampling.

\section{Comparison of plasma $\mathrm{CRH}, \mathrm{CRP}$, progesterone and 17-hydroxyprogesterone concentrations by treatment group}

Table 2 compares plasma CRH, CRP, progesterone, 17-hydroxyprogesterone concentrations at each of the two epochs and the rate of change of these hormones between the two 
sampling times according to treatment group. The plasma concentrations of 17-OHPC at each epoch are also provided.

$\mathrm{CRH}$ concentrations and the rate of change of $\mathrm{CRH}$ concentration were not significantly different. CRP was significantly higher in epoch1 in the 17-OHPC group when compared with placebo. At epoch 2 however, plasma CRP concentrations were similar in the two treatment groups. Progesterone and 17-hydroxyprogesterone concentrations were not significantly different at epoch 1 and 2 between the 17-OHPC and placebo groups and neither were the rates of change. Concentrations of 17-OHPC increased slightly from epoch 1 to epoch 2 . The drug was not detected in women receiving placebo.

\section{Relationship of 17-OHPC with selected biomarkers of preterm birth}

We evaluated the relationship between 17-OHPC concentration and CRH, CRP, progesterone and 17-hydroxy-progesterone. Table 3 summarizes these relationships. A highly significant positive monotonic relationship is seen between 17-OHPC concentration and progesterone concentration as well as 17-OHPC concentration and 17hydroxyprogesterone at both sample times. These relationships at epoch 2 are depicted in Figure 1 for progesterone and its metabolite 17-hydroxyprogesterone. The rate of change in progesterone concentration likewise is positively related to the concentration of 17-OHPC.

Despite the fact that CRP was significantly higher in the 17-OHPC group compared with the placebo group in epoch 1, we could not demonstrate a concentration relationship between 17-OHPC and CRP either at epoch 1 or epoch 2 or over the rate of change between epoch 1 and 2.

There was no correlation between CRH concentration and 17-OHPC concentration at epoch1; however, at epoch $2 \mathrm{CRH}$ was significantly correlated with the concentration of 17OHPC (Spearman's $\rho=0.31, p=0.02)$ as was the rate of rise of $\mathrm{CRH}(\rho=0.34, \mathrm{p}=0.01)$ and these relationships are depicted in Figure 2.

\section{Relationship of plasma 17-OHPC, CRH, CRP, progesterone and 17-hydroxy-progesterone to gestational age at delivery}

We evaluated the relationship of the five plasma analytes (17-OHPC, CRP, CRH, 17hydroxyprogesterone and progesterone) at the first sampling period to the outcome of gestational age at delivery in the placebo and treatment groups in a proportional hazards model, adjusting for confounders. The relationship between each of these analytes and gestational age of delivery is summarized in Table 4. In the placebo group no significant relationship was identified between the analytes and gestational age at delivery. Among the 17-OHPC treated group none of the analytes was significantly related to gestational age of delivery, except for 17-OHPC concentration which demonstrated a highly significant negative relation to gestational age at delivery. Figure 3 demonstrates the relationship between 17-OHPC concentration and gestational age at delivery. When adjusted for gestational age at the time of blood draw, race/ethnicity and BMI (Table 4) the hazard ratio was highly significant $\left(1.14, \mathrm{p}=0.001, \mathrm{R}^{2}=0.49\right)$.

We compared characteristics of those women who received all their injections with those who did not receive all their injections for reasons of non-compliance. We found no significant differences in maternal age, BMI, parity or number of prior preterm births. We also dichotomized 17-OHPC concentrations (above and below the 75th percentile ) to assure that those women with higher concentrations did not have some characteristic that might also be a risk factor for preterm birth. We found no significant difference between high 17ohpc concentration group ( $\geq 14 \mathrm{ng} / \mathrm{ml}$ ) and the lower concentration $(<14 \mathrm{ng} / \mathrm{ml}$ ) group in the 
frequency of potential risk factors of preterm birth like AA race, any prior preterm birth or monochorionic placentation.

\section{Discussion}

This is the first report of the relationship between plasma 17-OHPC concentrations and gestational age at delivery. ${ }^{7}$ The current study provides considerable insight into the drug's impact on selected markers of preterm birth and on endogenous hormones. The most intriguing findings of this study relate to the relationship of 17-OHPC and gestational age at delivery particularly with evidence of early systemic inflammation in women receiving 17 OHPC. Adjusting for 17-OHPC in the proportional hazards regression, as well as race/ ethnicity, BMI and gestational age at blood draw, CRP did not show a significant relationship with gestational age at delivery $(\mathrm{p}=0.06)$. In addition, we could not demonstrate a relationship between 17-OHPC and CRP concentrations suggesting that the negative relationship between gestational age at delivery and 17-OHPC concentrations may not be primarily attributable to an effect of the elevated CRP. Labor and parturition are recognized as inflammatory states; therefore, it is possible that the elevated CRP concentrations represent a concomitantly activated parturitional process rather than an antecedent cause of the parturitional process. Although CRP was significantly higher in the 17-OHPC treated group compared with the placebo group was significantly higher in the 17-OHPC treated group compared with the placebo group at 24-28 weeks, by 32-35 weeks the CRP concentrations were similar in the two groups. The apparent negative association of 17OHPC concentration and gestational age at delivery was not associated with significant difference in gestational age at delivery in parent study or in the ancillary study reported here $^{2}$. In the parent study, delivery or fetal death before 35 weeks occurred in $41.5 \%$ of pregnancies in the 17-OHPC group and $37.3 \%$ of those in the placebo group (relative risk, $1.1 ; 95 \%$ confidence interval [CI], 0.9 to 1.3 ). Yet, among these same subjects we were able to demonstrate a highly significant negative relationship between 17-OHPC concentrations and gestational age at delivery. A similar relationship is seen between 17-OHPC and progesterone concentrations. Although mean progesterone concentrations did not differ significantly between treatment groups a very strong relationship was seen between 17OHPC and progesterone concentrations. These observations suggest that the negative association between 17-OHPC concentration and gestational age at delivery may impact a limited subset of subjects with twin gestation. For example, women with specific progesterone receptor (PR) genotype polymorphisms may be at particular risk. Manuck et $\mathrm{al}^{9}$ reported that certain PR single nucleotide polymorphisms (SNPs) were associated with higher risk of preterm birth in response to 17-OHPC. Whether PR SNPs were responsible for the inverse relationship between 17-OHPC concentration and gestational age at delivery noted in this study is unknown as we did not determine PR polymorphisms in this group.

The inverse relationship between 17-OHPC concentration and gestational age at delivery and the elevated CRP in the 17-OHPC group in twins should not be extrapolated to singleton gestations since the drug concentration and pregnancy outcome relationships may be different. Clearly additional pharmacologic data may help to define the relationship between progesterone supplementation and pregnancy outcome in twins. Whether these relationships have applicability to singleton gestation is unknown.

We also found several interesting and perhaps clinically relevant associations with 17OHPC use. First we found a very strong relationship between 17-OHPC and progesterone concentrations even though plasma progesterone concentrations were, overall, not significantly different between the two treatment groups. This can be explained by the fact that several factors affect progesterone concentrations and the impact of 17-OHPC on plasma progesterone concentration is relatively small compared with these other factors. In 
human liver microsomes, progesterone competes with metabolism of 17-OHPC. ${ }^{10}$ Progesterone and 17-OHPC are both metabolized by CYP $3 \mathrm{~A} 4$ and it is likely that the increase in progesterone concentrations is due to some degree of inhibition with the metabolizing enzyme. ${ }^{11}$ In monkeys, $17-\mathrm{OHPC}$ clearly increases progesterone concentrations ${ }^{12}$ but in human studies, including the current one, median values are increased with 17-OHPC treatment but only modestly. ${ }^{13,14}$ Whether the increase in progesterone concentrations has a benefit on pregnancy outcome cannot be determined at this time. The fact that progesterone supplementation seems to reduce preterm birth in certain subsets of at-risk women suggests that an increase in progesterone concentrations is central to any beneficial effect of progestin supplementation. Plasma progesterone concentrations do not reflect tissue concentrations of progesterone thus the positive impact of 17-OHPC on progesterone concentration may be much greater at the tissue level than at the plasma level and may account for any benefit attributable to this therapy.

The strength of the relationship between CRH and 17-OHPC concentration is relatively modest and, in fact, 17-OHPC is positively not negatively associated with CRH concentration. It is important to evaluate the relationship between CRH and 17-OHPC because CRH increases in both term and preterm births weeks prior to delivery. Since 17OHPC is positively related to CRH concentration, this could imply a potential adverse effect of 17-OHPC treatment. Although the dose relationship is weak, it is possible that 17-OHPC has a clinically relevant impact on CRH and gestational age at delivery. In fact, we have previously demonstrated that although BMI has a very modest relationship $(\mathrm{r}=-0.28$ to -0.34) to 17-OHPC concentration, when BMI is modeled against plasma 17-OHPC concentrations, it has a very dramatic effect on plasma concentrations ${ }^{15}$. Clearly additional data are required to reach any meaningful conclusion about this intriguing relationship especially in singleton gestation.

In conclusion, we have demonstrated, for the first time, the relationship between 17-OHPC concentration and gestational age at delivery in women with twin gestation. This may define more clearly the potential risks and benefits of this therapy. We have demonstrated that among women with twin gestation higher plasma concentrations of 17-OHPC are associated with a shorter gestational age at delivery than lower concentrations of 17-OHPC. Women receiving 17-OHPC have higher CRP values than women receiving placebo but no dose relationship between CRP and 17-OHPC is apparent. We have also demonstrated a strong dose-relationship between 17-OHPC and progesterone and a modest but significant relationship between 17-OHPC and CRH especially the rate of rise of CRH. The findings above apply to women with twin gestation and cannot be assumed to pertain in women with singleton gestation. More research is required to define the mechanism of action of 17OHPC and to optimize the dose of 17-OHPC especially in singleton gestation where efficacy has been demonstrated. An urgent need exists to identify subsets of women who may most benefit from this therapy and to define those who might be harmed by this therapy.

\section{Acknowledgments}

The authors thank the following protocol subcommittee members who participated in protocol development and coordination between clinical research centers (Margaret Cotroneo, R.N. and Allison T. Northen, M.S.N., R.N.), and protocol/data management and statistical analysis (Elizabeth Thom, Ph.D.).

The project described was supported by grants from the Eunice Kennedy Shriver National Institute of Child Health and Human Development (HD27869, HD21410, HD40512, HD34136, HD34208, HD40485, HD27915, HD40544, HD40560, HD27917, HD40500, HD34116, HD40545, HD27860, HD3680) and does not necessarily represent the official views of the NICHD or the National Institutes of Health. 


\section{References}

1. Meis PJ, Klebanoff M, Thom E, et al. for the NICHD Maternal-Fetal Medicine Network. Prevention of recurrent preterm delivery by 17 alpha-hydroxyprogesterone caproate. N Engl J Med. 2003; 348:2379-85. [PubMed: 12802023]

2. Rouse DJ, Caritis SN, Peaceman AM, et al. for the NICHD Maternal-Fetal Medicine Units Network. A trial of 17 alpha-hydroxyprogesterone caproate to prevent prematurity in twins. N Engl J Med. 2007; 357:454-61. [PubMed: 17671253]

3. Petraglia F, Imperatore A, Challis JRG. Neuroendocrine Mechanisms in Pregnancy and Parturition. Endocr Rev. 2010; 31:783-816. [PubMed: 20631004]

4. Chaisilwattana P, Monif GRG. Potential Use of C-Reactive Protein Determinations in Obstetrics and Gynecology. Obstet Gynecol Surv. 1989; 44:355-360. [PubMed: 2657521]

5. Zhang S, Mada S, Sharma S, et al. Identification of Enzymes Involved in the Simultaneous quantitation of 17a-hydroxyprogesterone caproate, 17a-hydroxyprogesterone and progesterone in humans using high performance liquid chromatography-mass spectrometry( HPLC-MS/MS). Journal of Pharmaceutical and Biomedical Analysis. 2008; 48:1174-1180. [PubMed: 18947956]

6. Linton EA, Perkins AV, Hagan P, et al. Corticotrophin-releasing hormone (CRH)-binding protein interference with CRH antibody binding: implications for direct CRH immunoassay. J Endocrinol. 1995; 146:45-53. [PubMed: 7561620]

7. Caritis SN, Shringi S, Venkataramanan R. Pharmacokinetics of 17-hydroxyprogesterone caproate in multifetal gestation. Am J Obstet Gynecol. 2011; 205:40.e1-8. [PubMed: 21620357]

8. Pubmed, June 1954-Aug23, 2011, English language journals, keywords: delalutin, hexanoate, 17-P, 17-OHPC, 17 hydroxyprogesterone caproate, 17 hydroxyprogesterone hexanoate

9. Manuck TA, Lai Y, Meis PJ, et al. for the NICHD Maternal-Fetal Medicine Network. Progesterone receptor polymorphisms and clinical response to 17-alpha-hydroxyprogesterone caproate. Am J Obstet Gynecol. 2011; 205:135.e1-9. [PubMed: 21600550]

10. Cuppett C, Zhao W, Caritis S, et al. Effects of endogenous steroids on 17 alphahydroxyprogesterone caproate (17-OHPC) metabolism. Am J Obstet Gynecol. 2011; 204:S29.

11. Sharma S, Ou J, Strom S, Mattison D, et al. Identification of Enzymes Involved in the Metabolism of 17-Hydroxyprogesterone Caproate: An Effective Agent for Prevention of Preterm Birth. Drug Metab Dispos. 2008; 36:1896-1902. [PubMed: 18573861]

12. Aksel S, Yeoman R, Hazelton J, et al. The Effects of 17-hydroxyprogesterone Caproate (17OHPC) Administration to Pregnant Squirrel Monkeys (Saimiri boliviensis boliviensis). Am J Primatol. 2005; 25:175-183.

13. Hartikainen-Sorri AL, Kauppila A, Tuimala R. Inefficacy of 17-hydroxyprogesterone caproate in the Prevention of Prematurity in Twin Pregnancy. Obstet Gynecol. 1980; 56:692-5. [PubMed: 7443111]

14. Johnson JW, Lee PA, Zachary AS, et al. High-risk prematurity--progestin treatment and steroid studies. Obstet Gynecol. 1979; 54:412-8. [PubMed: 492618]

15. Caritis SN, Sharma S, Venkataramanan R, et al. Pharmacokinetics of 17-hydroxyprogesterone caproate in multifetal gestation. Am J Obstet Gynecol. 2011; 205:40.e1-8. [PubMed: 21620357]

\section{Appendix}

In addition to the authors, other members of the Eunice Kennedy Shriver National Institute of Child Health and Human Development Maternal-Fetal Medicine Units Network are as follows:

University of Pittsburgh - E. Daugherty, M. Cotroneo, R. Venkataramanan, S. Zhang

University of Alabama at Birmingham - W. Andrews, J. Sheppard, A. Northen

Northwestern University - M. Dinsmoor (NorthShore University Health System), G. Mallett, P. Simon, M. Huntley, M. Ramos 
Drexel University - M. Hoffman, S. Wilson, C. Tocci, M. Lake, M. Talucci

University of Utah - K. Anderson, F. Porter (LDS Hospital), A. Guzman (McKay-Dee Hospital Center), K. Jolley (Utah Valley Regional Medical Center), S. Quinn (LDS Hospital)

Columbia University — R. Berkowitz, S. South, L. Paley, S. Bousleiman, V. Carmona

The Ohio State University -F. Johnson, C. Latimer

Case Western Reserve University-MetroHealth Medical Center-C. Milluzzi, C. Heggie, H. Ehrenberg, B. Stetzer, A. Merlino

University of North Carolina at Chapel Hill - K. Boggess, K. Dorman, S. Timlin

Wayne State University — G. Norman, C. Sudz, S. Blackwell

Brown University - D. Allard

University of Texas Southwestern Medical Center, Dallas - K. Leveno, L. Moseley

University of Texas Health Science Center at Houston - D. Soebbing-Cross, J. Martinez, B. Glenn-Cole, L. Gilstrap

Wake Forest University Health Sciences - P. Meis, M. Swain, K. Johnson, K. Lanier, C. Leftwich

The George Washington University Biostatistics Center - E. Thom, A. Braga, E. Cardenas, L. Leuchtenburg

Eunice Kennedy Shriver National Institute of Child Health and Human Development -S. Tolivaisa 
A.

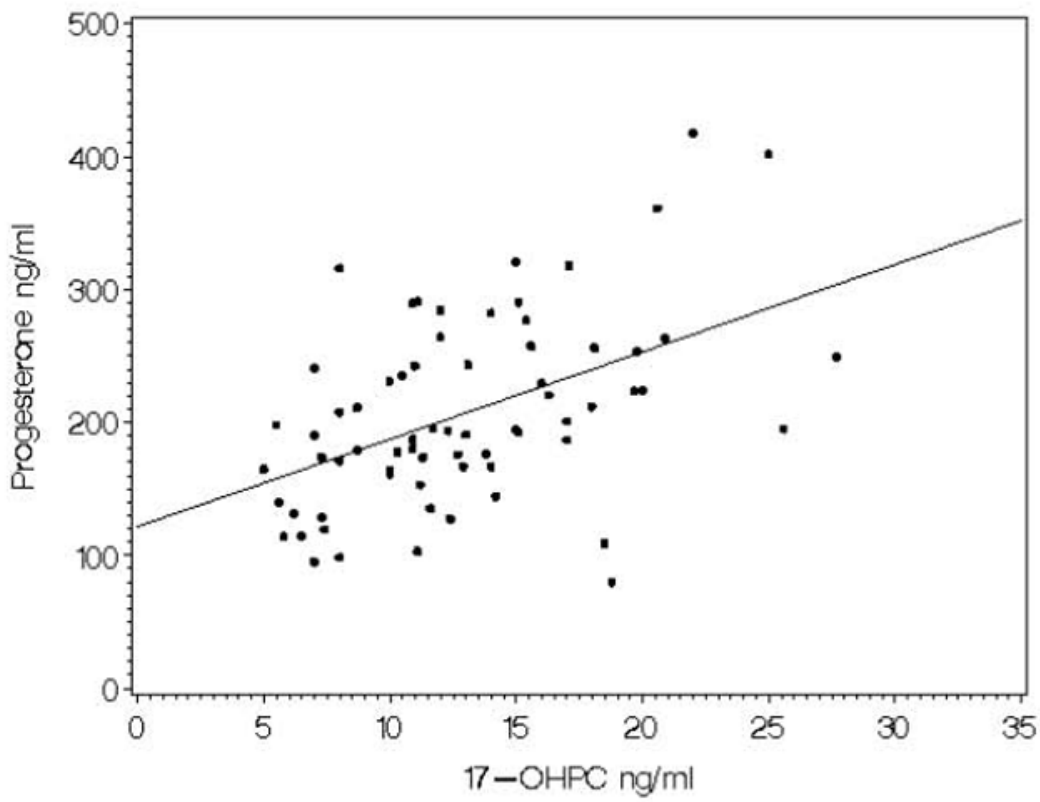

B.

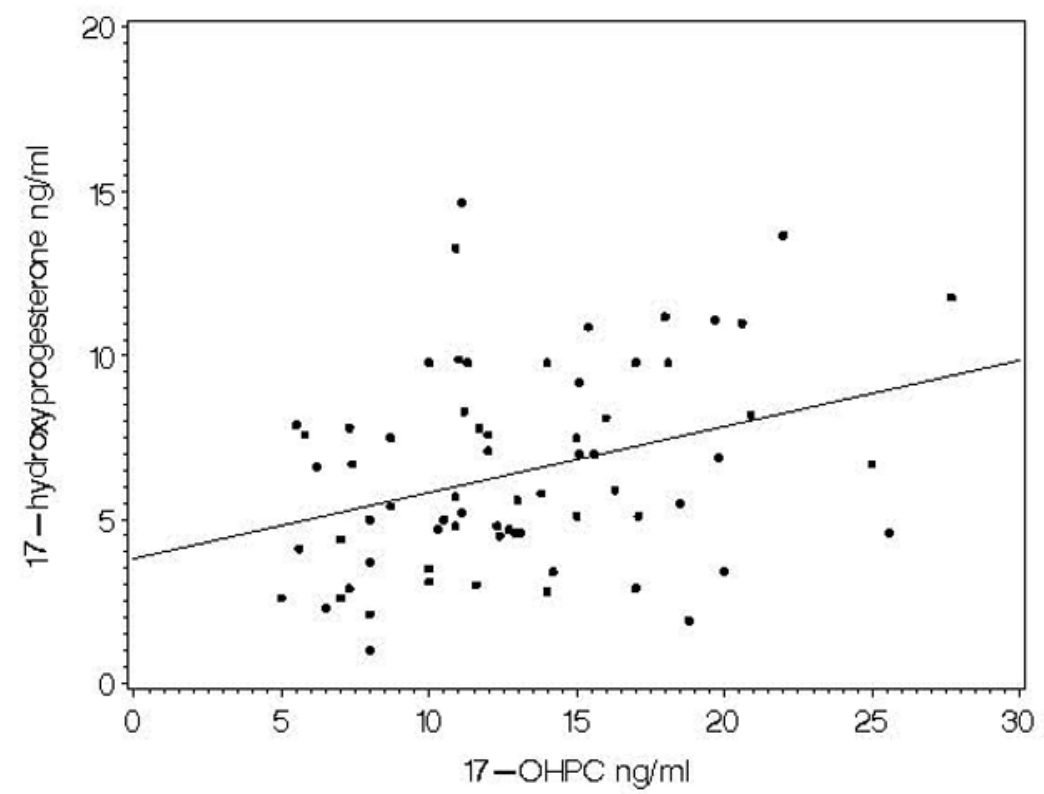

Figure 1.

Relationship of 17-OHPC concentration with progesterone and 17-hydroxyprogesterone in epoch 2. Spearman correlation coefficient $=0.46(\mathrm{p}<0.0001)$ for 17-OHPC vs progesterone and Spearman correlation coefficient $=0.29(\mathrm{p}=0.01)$ for 17-OHPC vs 17-

hydroxyprogesterone. 
A.

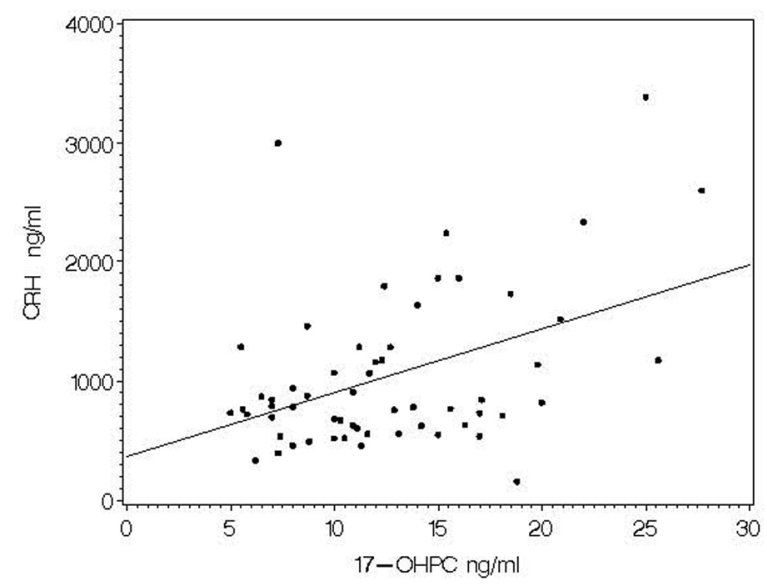

B.

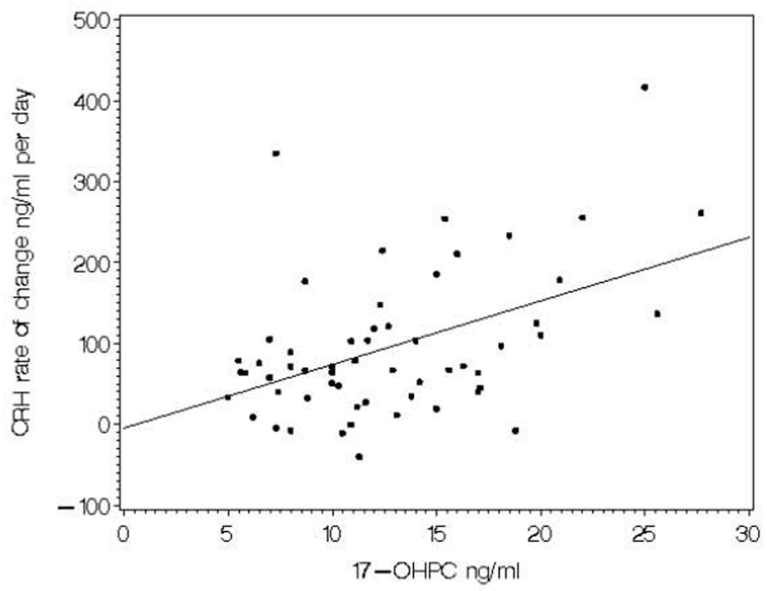

Figure 2.

Relationship of 17-OHPC to CRH concentration in epoch 2 and to the rate of change of CRH. Spearman correlation coefficient $=0.31(\mathrm{p}=0.02)$ for $17-\mathrm{OHPC}$ vs CRH concentration in epoch 2 and Spearman correlation coefficient $=0.34(\mathrm{p}=0.01)$ for $17-\mathrm{OHPC}$ vs rate of change of CRH. 


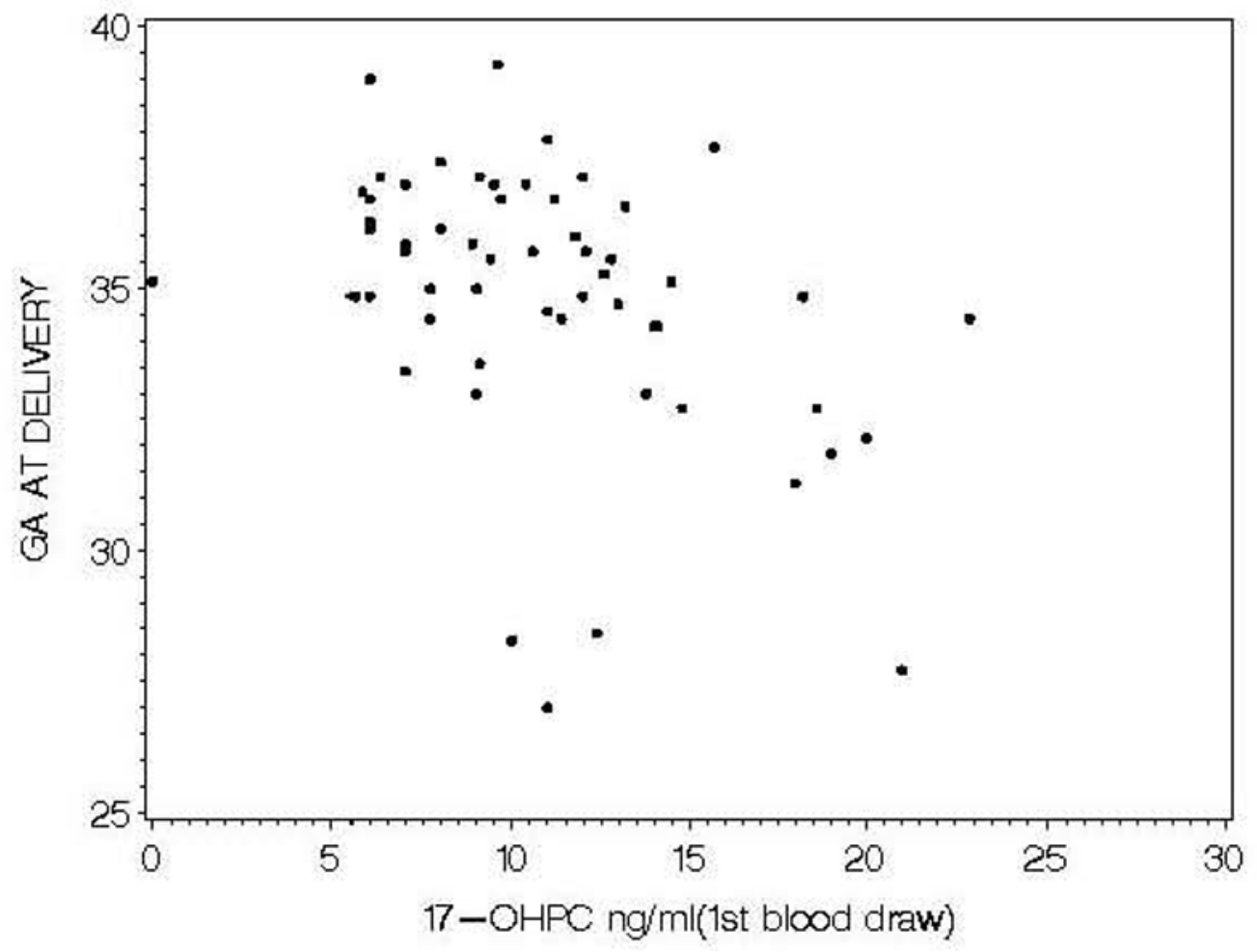

Figure 3.

Scatterplot of 17-OHPC concentration and gestational age at delivery. 


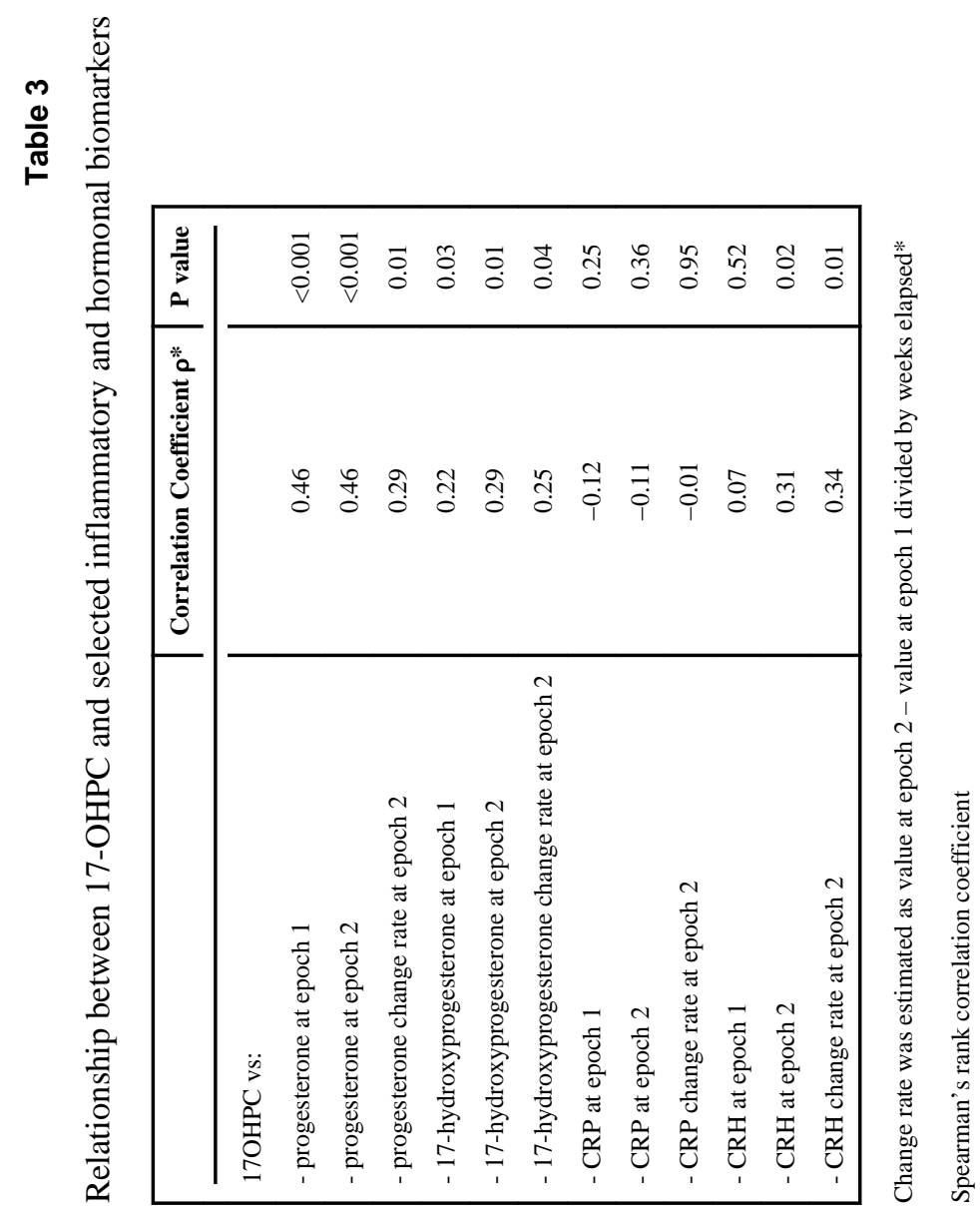

Am J Obstet Gynecol. Author manuscript; available in PMC 2013 November 01. 


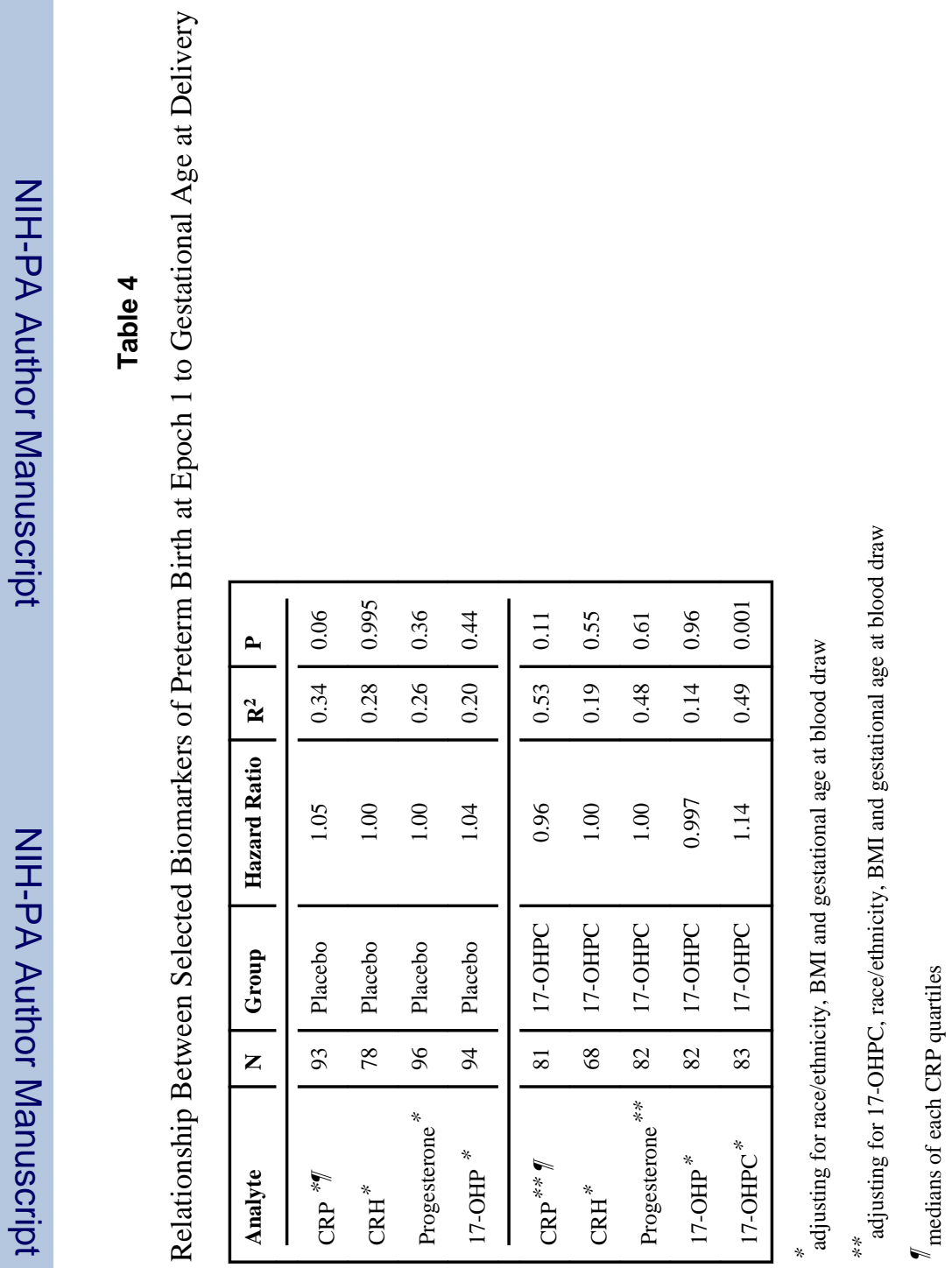

Am J Obstet Gynecol. Author manuscript; available in PMC 2013 November 01. 